\title{
An intraline of conical intersections for methylamine
}

\author{
C. Levi, ${ }^{1}$ G. J. Halász, ${ }^{2}$ Á. Vibók, ${ }^{3}$ I. Bar, ${ }^{1}$ Y. Zeiri, ${ }^{4}$ R. Kosloff, ${ }^{5}$ and M. Baer ${ }^{6, a)}$ \\ ${ }^{1}$ Department of Physics, Ben-Gurion University, Beer Sheva 84105, Israel \\ ${ }^{2}$ Department of Information Technology, University of Debrecen, P.O. Box 12, H-4010 Debrecen, Hungary \\ ${ }^{3}$ Department of Theoretical Physics, University of Debrecen, P.O. Box 5, H-4010 Debrecen, Hungary \\ ${ }^{4}$ Department of Biomedical Engineering, Ben-Gurion University, Beer Sheva 84105, Israel \\ ${ }^{5}$ Department of Physical Chemistry and the Fritz Haber Center for Molecular Dynamics, The Hebrew \\ University of Jerusalem, Jerusalem 91904, Israel \\ ${ }^{6}$ The Fritz Haber Center for Molecular Dynamics, The Hebrew University of Jerusalem, \\ Jerusalem 91904, Israel
}

(Received 20 March 2008; accepted 19 May 2008; published online 23 June 2008)

In this article are considered the conical intersections ( $c i$ 's) related to the $\mathrm{N}-\mathrm{H}$ bond in the methylamine, $\mathrm{CH}_{3} \mathrm{NH}_{2}$, molecule. The novel feature that was revealed is that the two lowest states $1 A^{\prime}$ and $1 A^{\prime \prime}$ are coupled by a line of cis located in $\mathrm{HC}-\mathrm{NHH}$ plane-a line that is formed by moving a single hydrogen on that plane while fixing the (six) other atoms. The validity of this line was proven first by studying the singularities of the (angular) nonadiabatic coupling terms and then by revealing the degeneracy points formed by the two interacting adiabatic potential energy surfaces (PESs). A theoretical analysis indicated that the line has to be a finite closed line. We also calculated the Berry phase for a contour that surrounds this line and found it to be $3.127 \mathrm{rad}$, namely, a value reasonably close to $\pi$. The existence of such lines of cis-instead of isolated cis (as exhibited by other $n$-atomic $(n>3)$ molecules such as $\mathrm{HNCO}$ or $\left.\mathrm{C}_{2} \mathrm{H}_{2}\right)$-may enhance significantly the transition rate from an upper adiabatic state to a lower one. There are also numerical advantages in such situations, that is, if such a line is properly placed in that plane (like in the present case) the wave-packet treatment of the nuclei can be carried out employing a single diabatic PES instead of having to consider two coupled PESs. () 2008 American Institute of Physics.

[DOI: $10.1063 / 1.2943143$ ]

\section{INTRODUCTION}

The study of the electronic nonadiabatic coupling terms (NACTs) and conical intersections $(\mathrm{ci})$ concentrated, for the past two decades, mainly on small molecules, namely, triatomic and tetra-atomic systems. ${ }^{1-17}$ In the present article we consider a much larger system, the methylamine molecule, $\mathrm{CH}_{3} \mathrm{NH}_{2}$, consisting of seven atoms. The structure of the molecule is given in Fig. 1(a) where it can be seen that the methyl group, $\mathrm{CH}_{3}$, is separated from the amine group, $\mathrm{NH}_{2}$ by the $\mathrm{CN}$ bond. This molecule has long been a question of considerable interest since it comprises two strongly coupled large amplitude motions, specifically, the torsion of the methyl top and the inversion of the amine group. ${ }^{17}$ Several experimental studies provided evidence that the dominant channel in its photodissociation in the first absorption band corresponds to the $\mathrm{N}-\mathrm{H}$ bond fission. ${ }^{18-20}$ Very recently ${ }^{21-23}$ it was shown that in the vibrational mediated photodissociation of $\mathrm{CD}_{3} \mathrm{NH}_{2}$ about $90 \%$ of the total observed products (only hydrogen isotopes were measured) are $\mathrm{H}$ photofragments released from the amine group. Also, theoretical findings showed the cuts through the $a b$ initio potential energy surfaces (PESs) for the ground $1 A^{\prime}$ and the first excited $1 A^{\prime \prime}$ states of methylamine. Particularly, it was pointed out that the $1 A^{\prime \prime}$ state potential that leads to the breakup of the $\mathrm{N}-\mathrm{H}$ bond is characterized by a small barrier $\left(\sim 3000 \mathrm{~cm}^{-1}\right)$ at

\footnotetext{
${ }^{a)}$ Electronic mail: michaelb@fh.huji.ac.il.
}

short range and is followed, at larger bond extensions, by a $c i$ located at an intersection point with the $1 A^{\prime}$ state where both states belong to the $C_{s}$ symmetry. ${ }^{24,25}$

In what follows we report on a study of NACTs that couple the above mentioned two states and briefly refer to the higher excited state, $2 A^{\prime \prime}$.

\section{FORMING THE INTRALINE OF CONICAL INTERSECTIONS}

In what follows we report on a study of NACTs that couple the above mentioned three states. To carry out this study the triatomic amine group is assumed to form a plane with $\mathrm{HC}$ diatom of the methyl group (see Fig. 1), and our study concentrates on searching for Jahn-Teller cis (Refs. 26-28) between the three lower states, namely, the ground $1 A^{\prime}$ state and the next two excited states $1 A^{\prime \prime}$ and $2 A^{\prime \prime}$ (the search for these cis is carried out by allowing the amine hydrogen to move in this plane only).

To this end we employ the MOLPRO package ${ }^{29}$ with the multireference configuration interaction method and the $6-31+\mathrm{G}^{*}$ basis set. We used the active space, including two valence electrons distributed on five orbitals (three of them belonging to the irreducible representation of $A^{\prime}$ ). Three electronic states were computed with equal weights. All seven atoms were considered, but the search for cis is carried out by activating only one atom-an amine hydrogen-as a test particle. To follow the motion of the hydrogen we em- 
(a) (b)

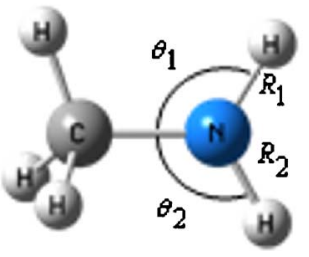

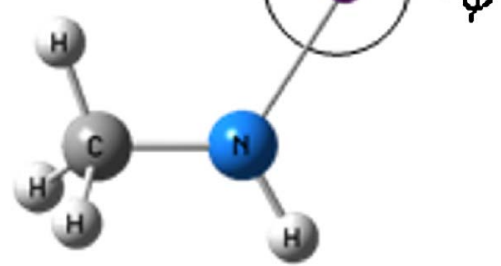

FIG. 1. (Color online) The equilibrium structure of methylamine. In the present study five atoms, namely, the carbon, the nitrogen, two amine hydrogens, and a methyl hydrogen are assumed to form a fixed plane-the $\mathrm{HC}-\mathrm{NH}_{2}$ plane - and their relative motion is constrained to this plane. (a) The coordinates $\theta_{1}$ and $R_{1}$ show the position of the test hydrogen (with respect to the nitrogen) that were varied during the $c i$ search (the coordinates $\theta_{2}$ and $R_{2}$ are held fixed during this process). (b) The polar coordinates $q$ and $\varphi$ show the position of test hydrogen with respect to an assumed point close to a $c i$ point.

ploy two (polar) coordinates, the radial coordinate $q$ and the angular coordinate $\varphi$, defined for a system of coordinates located at some point in the plane [see Fig. 1(b)]. The detection is done by calculating the angular NACT, $(1 / q) \tau_{\varphi j k}(\varphi, q \mid \mathbf{s})$, where $\tau_{\varphi j k}(\varphi, q \mid \mathbf{s})$ is defined as

$$
\tau_{\varphi j k}(\varphi, q \mid \mathbf{s})=\left\langle\zeta_{j}\left(\mathbf{s}_{e} \mid \varphi, q, \mathbf{s}\right) \mid \frac{\partial}{\partial \varphi} \zeta_{k}\left(\mathbf{s}_{e} \mid \varphi, q, \mathbf{s}\right)\right\rangle .
$$

Here $\zeta_{i}\left(\mathbf{s}_{e} \mid \varphi, q, \mathbf{s}\right) ; i=j, k$ are the electronic (adiabatic) BornOppenheimer (BO) eigenfunctions, ${ }^{30,31} \mathbf{s}_{e}$ stands for the set of electronic coordinates, and $\mathbf{s}$ presents the group of all nuclear coordinates excluding $\varphi$ and $q$. Having defined the system of coordinates the search starts by forming circular contours of different radii and calculating the angular NACTs along these contours. The next step is deriving the adiabaticto-diabatic transformation (ADT) angle (known also as the mixing angle), $\gamma(\varphi, q)$ (Ref. 32) (see also Ref. 1, Chap. 3.1),

$$
\gamma(\varphi, q \mid \mathbf{s})=\int_{0}^{\varphi} d \varphi^{\prime} \boldsymbol{\tau}_{\varphi}\left(\varphi^{\prime}, q \mid \mathbf{s}\right) .
$$

In the case of a closed contour we get $\gamma(\varphi=2 \pi, q \mid \mathbf{s})$ $=\alpha(q \mid s)$, where $\alpha(q \mid s)$ is recognized as the Berry phase. ${ }^{33,34}$ It is important to emphasize that for situations where the NACT is formed, in a given region, by two isolated states (namely, states affected, at most, slightly by higher states in that region), the phase $\alpha(q \mid \mathbf{s})$ is expected to be close to an integer multiple of $\pi$ (or zero). ${ }^{33-36}$ Part of the numerical study was devoted to this issue. We calculated NACTs between the three lowest states of the $C_{s}$ symmetry, namely, $1 A^{\prime}, 1 A^{\prime \prime}$, and $2 A^{\prime \prime}$ (mentioned earlier) and found that only the NACTs formed by the two lower states are nonzero whereas the other two that involve the second excited state are practically zero (thus guaranteeing the isolation of the two lower states in the region of interest).

Figure 2(a) presents the angular NACT, along such a circle, as a function of $\varphi$ with $q=0.1 \AA$, where the coordinates of the center are at $\left(R_{1}, \theta_{1}\right)=\left(1.83 \AA, 122^{\circ}\right)$ and $\left(R_{2}, \theta_{2}\right)=\left(1.0 \AA, 120^{\circ}\right)$ [see Fig. 1(a)]. The figure shows two relatively sharp peaks with opposite signs. It turns out that the value of the integral under each peak is $\sim 1.554 \mathrm{rad}$ (i.e.,
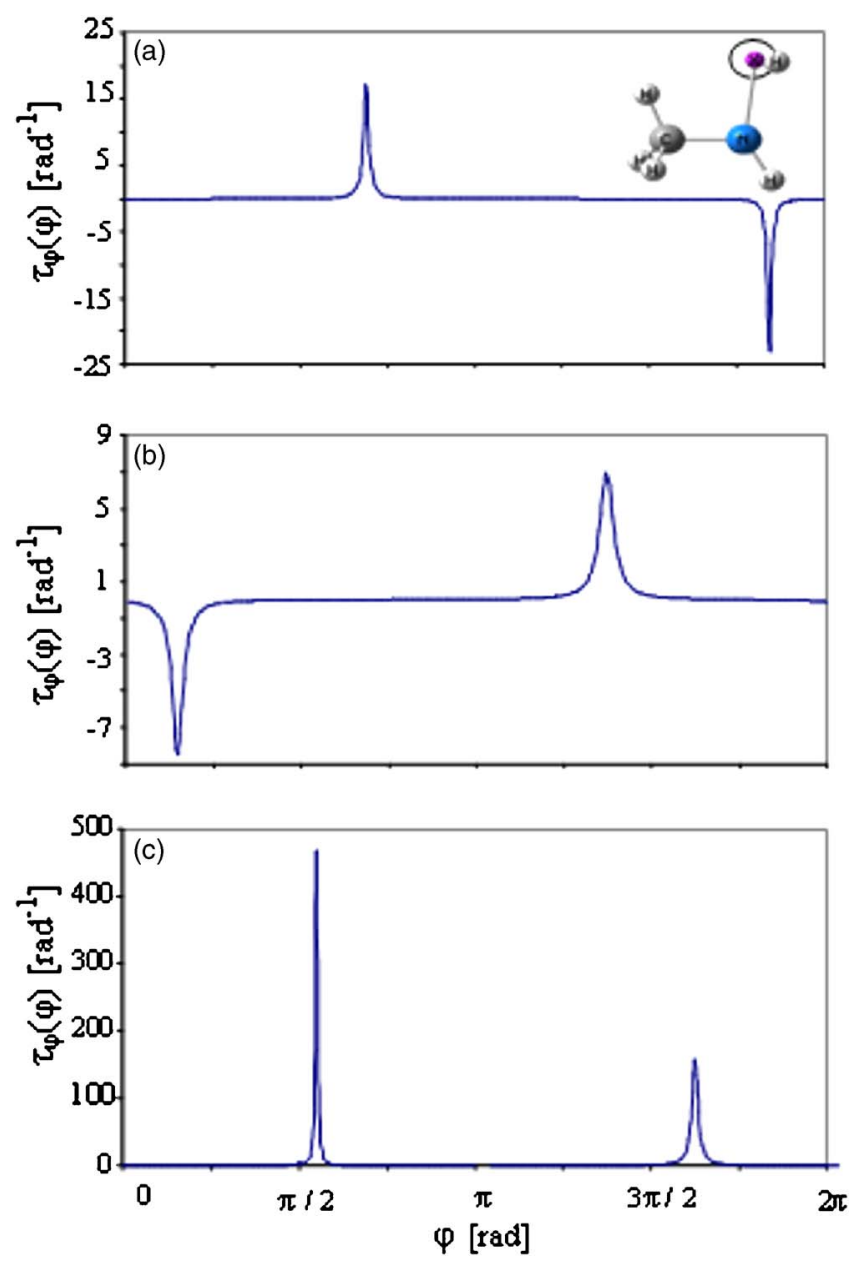

FIG. 2. (Color online) The angular NACT presented as a function of $\varphi$ for $q=0.1 \AA$ as calculated at three different locations along the seam (see Fig. 3): (a) The NACT at point $\left(R_{1}, \theta_{1} ; R_{2}, \theta_{2}\right)=\left(1.83 \AA, 122^{\circ} ; 1.0 \AA, 120^{\circ}\right)$. (b) The NACT at point $\left(R_{1}, \theta_{1} ; R_{2}, \theta_{2}\right)=\left(1.70 \AA, 85^{\circ} ; 1.0 \AA, 120^{\circ}\right)$. (c) The NACT at point $\left(R_{1}, \theta_{1} ; R_{2}, \theta_{2}\right)=\left(1.70 \AA, 159^{\circ} ; 1.0 \AA, 120^{\circ}\right)$.

a value close to $\pi / 2$ ) and the value of the Berry phase is $\alpha(q \mid \mathbf{s}) \sim 0$. It is important to emphasize that the structure of the angular NACT differs significantly from all other angular NACTs for which we usually encounter two ${ }^{1,4(b), 4(c), 5,6,7(a), 8,28}$ (and sometimes three ${ }^{4(\mathrm{a})}$ ) positive maximal values and the Berry phase becomes, approximately, an integer multiple of $\pi$ (and not zero).

Thus the question is what makes this particular case so different? Following additional calculations we find that the same plane hosts many more cis. All are characterized by the same feature as discussed above; namely, the corresponding angular NACTs posses two sharp peaks of opposite signs (see another one given in Fig. 2(b) with $q=0.1 \AA$ and the center at $\left[\left(R_{1}, \theta_{1}\right)=\left(1.70 \AA, 85^{\circ}\right)\right]$. Finally it was established that the just mentioned cis form a continuous (finite) line (see Fig. 3). This line is constructed in practice by connecting all the positive (or negative) peaks of NACTs of the type presented in Figs. 2(a) and 2(b). It is interesting to note that one of the $c i$ points, i.e., the one calculated at $R_{1}=1.83 \AA$ and $\theta_{1}=122^{\circ}$, is close (but not identical) to the position of a degeneracy point revealed sometime ago by Dunn and Morokuma. ${ }^{24}$

In Fig. 3 is presented such a line in a plane determined 


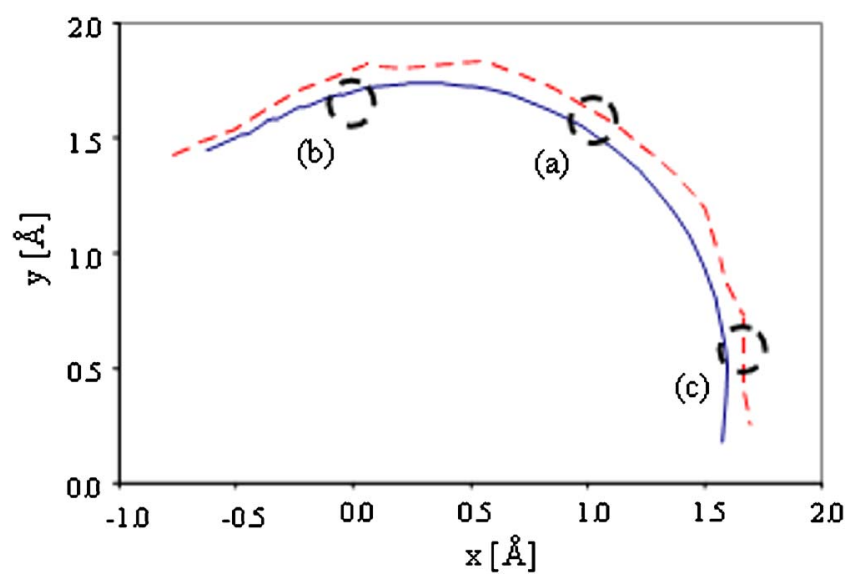

FIG. 3. (Color online) The intra (fixed configuration space) seam for the ci's located in the $\mathrm{HC}-\mathrm{NH}_{2}$ plane as calculated for $R_{2}=1.0 \AA$ and $\theta_{2}=120^{\circ}$ : (---) The seam as calculated by connecting the $c i$ points; (----) the seam as calculated by connecting the degeneracy points. The two Cartesian coordinates $(x, y)$ are related to the polar coordinates $\left(R_{1}, \theta_{1}\right)$, namely, $(x, y)$ $=\left[R_{1} \cos \left(\pi-\theta_{1}\right), R_{1} \sin \left(\pi-\theta_{1}\right)\right]$. At three locations are drawn small circles to indicate the positions of three contours mentioned in Fig. 2.

by holding the second amine hydrogen fixed at $\left(R_{2}, \theta_{2}\right)$ $=\left(1.0 \AA, 120^{\circ}\right)$ [see Fig. 1(a)]: This line is presented in terms of Cartesian coordinates $(x, y)\left[=R_{1} \cos \left(\pi-\theta_{1}\right)\right.$, $R_{1} \sin \left(\pi-\theta_{1}\right)$ ], where the center of the coordinates is located at the nitrogen atom.

This just described line can be interpreted as a seam (defined as the line that connects all these infinite number of ci points).

Like any other seam this one is also expected to be a line that connects the degeneracy points between the two adiabatic surfaces. In Fig. 3 is presented a second line that follows the degeneracy points and closely overlaps with the seam (the deviations are within the error limit of the calculations).

More details concerning this line of degeneracy points are given in Fig. 4. In Fig. 4(a) are presented the two interacting adiabatic PESs $1 A^{\prime}$ and $1 A^{\prime \prime}$, assigned as $V_{1}(x, y)$ and $V_{2}(x, y)$. In Fig. $4(\mathrm{~b})$ is presented $\Delta V(x, y)$, the difference $\Delta V(x, y)=V_{2}(x, y)-V_{1}(x, y)$ between the two adiabatic PESs.

The two lines in the $x-y$ plane in Fig. 3 are seen to be finite and open. In fact additional calculations based on the NACTs to find out more about these lines failed altogether because the NACTs became spikier to the extent that finally we lost them altogether. However additional calculations based on degeneracy points were carried out, and they showed that, undoubtedly, the line is extending toward the two hydrogens - the amine hydrogen on one side and the corresponding methyl hydrogen on the other. Thus, this type of calculations seems to indicate that the line is finite. However we could not establish whether the line is open or closed mainly because if closed-the missing segment(s) is (are) at the high potential region where the accuracy is not good enough to establish points of degeneracy. These issues are further discussed in the next chapter.

More about the PESs of this system is shown in Fig. 5. In contrast to the adiabatic surfaces in Fig. 4(a) these are the corresponding diabatic ones. This figure clearly shows the important advantage in having a line of degeneracy points in
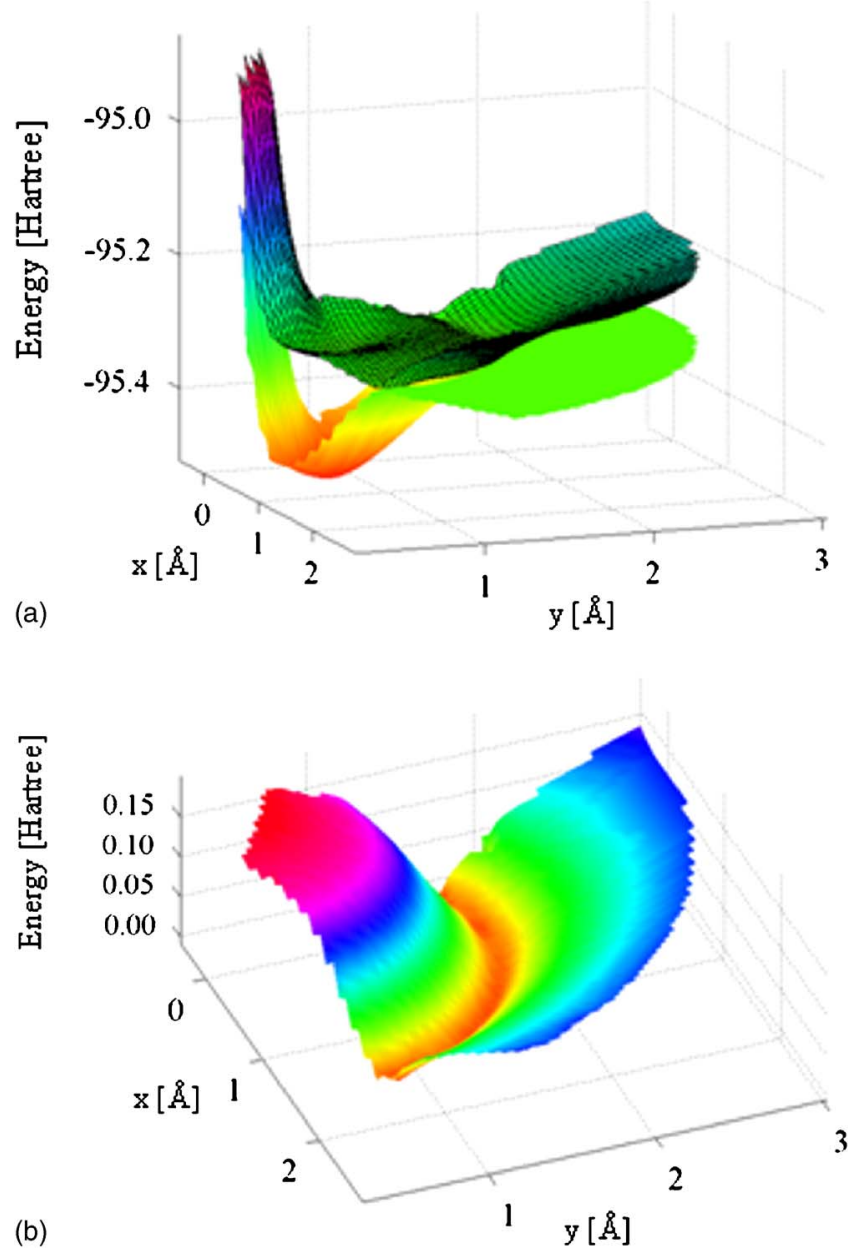

FIG. 4. (Color online) The PESs as a function of $x$ and $y$ as calculated for $R_{2}=1.0 \AA$ and $\theta_{2}=120^{\circ}$ : (a) Presents the two adiabatic $\left(1 A^{\prime}\right.$ and $\left.1 A^{\prime \prime}\right)$ PESs, $V_{1}(x, y)$ and $V_{2}(x, y)$; (b) presents the difference $\Delta V(x, y)=V_{2}(x, y)$ $-V_{1}(x, y)$ between the two PESs. The two figures are shown at different angles to emphasize the more significant features in each case.

a fixed plane. This situation causes the corresponding diabatic coupling term to be zero along this line, ${ }^{37,38}$ thus allowing a wave packet to move, freely and undisturbed, from the upper adiabatic surface to the lower adiabatic surface (more on this issue is discussed in the next chapter).

\section{DISCUSSION AND CONCLUSIONS}

This article concentrates on an exceptional continuous line of cis revealed in the methylamine molecule. This line can be interpreted as a seam, but if it is a seam it is less common because some of its features are different. In what follows we concentrate on several issues:

(1) In all previous studies ${ }^{1-8,14,15,28,34}$ the seams are located outside the planes (e.g., the $g$ - $h$ plane, which is defined as the perpendicular plane to the seam $\left.{ }^{7(a)}\right)$ that contain the moving test particle (which in the present case is the $\mathrm{HC}-\mathrm{NHH}$ plane). In other words the methylamine creates a phenomenon where the two interacting states i.e., $1 A^{\prime}$ and $1 A^{\prime \prime}$, are coupled by a continuous line of cis formed by one single moving atom where all other (six) atoms of the molecule are fixed in configuration space. Since this hydrogen is moving within the HC- 


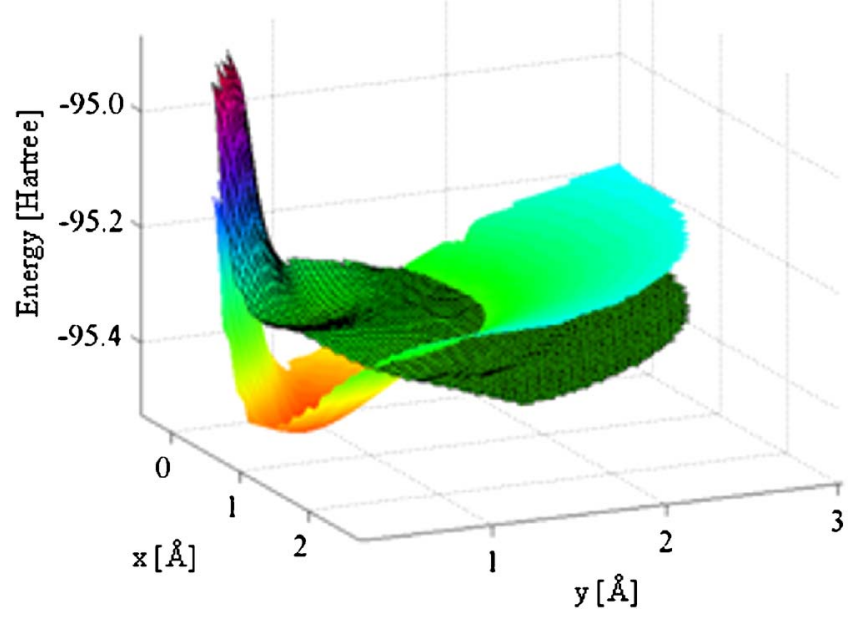

FIG. 5. (Color online) The two diabatic PESs as a function of $x$ and $y$ as calculated for $R_{2}=1.0 \AA$ and $\theta_{2}=120^{\circ}$. In the figure is emphasized the (physical) diabatic potential, which leads in a continuous way from the upper adiabatic PES to the lower one.

$\mathrm{NHH}$ plane, the line, just described, is located in that (HC-NHH) plane as well (see Fig. 3). As it stands this situation cannot be justified. To understand these findings we have to consider in more detail both the plane that contains the moving hydrogen and the $c i$-line as well as the two states that form the NACTs. It is well known that in order for two states (not coupled by any other state) to form degeneracy at a given point, two conditions have to be fulfilled, namely, ${ }^{38}$

$H_{11}-H_{22}=0$,

$H_{12}\left(=H_{21}\right)=0$,

where $H_{i j}, i, j=1,2$ are the matrix elements of the electronic Hamiltonian. For a randomly selected plane these conditions are at most satisfied at isolated points. However if a study is carried out with respect to a plane of symmetry (as in the present case) and the two states are of different symmetries (as indeed $1 A^{\prime}$ and $1 A^{\prime \prime}$ are), then condition (3b) is satisfied everywhere by symmetry. So now we are left with only one requirement to be satisfied, namely, Eq. (3a), and this one can be fulfilled along a line in the above mentioned plane without necessarily creating any improbable situations (as indeed is shown by the numerical treatment).

(2) Once we established the existence of the above mentioned $c i$-line - to be assigned as $\Lambda$-two questions have to be answered: (i) Is it a finite or an infinite line? (ii) If it is a finite line is it an open or a closed line? As indicated in the previous chapter we could not answer these questions numerically (although it is very likely that the line is indeed finite). In order to analyze the various possibilities we consider two kinds of contours, namely, $\Gamma_{c}$, that intersect $\Lambda$ and $\Gamma_{s}$, which surround it (see Fig. 6). As long as one moves along $\Gamma_{s}$ the order of the states $1 A^{\prime}$ and $1 A^{\prime \prime}$ at points $A$ and $B$ remains unchanged whereas if one follows a contour of the kind $\Gamma_{c}$ [see Fig. 6(a)], each crossing is accompanied with a
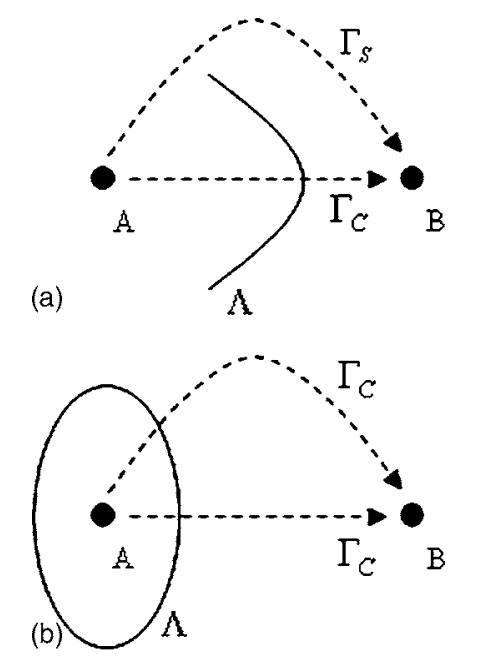

(c)

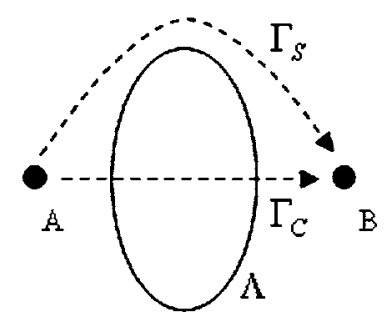

FIG. 6. A schematic picture showing contours $\Gamma$ intersecting finite $c i$ lines $\Lambda$ for various situations. $A$ and $B$ are two arbitrary points on the $x-y$ plane. (a) An open line $\Lambda$, a contour, $\Gamma_{c}$, that intersects it, and a contour, $\Gamma_{s}$, that surrounds it. (b) A closed line, $\Lambda$, point $A$ inside the surrounded area, point $B$ outside the surrounded area, and two contours $\Gamma_{c}$ that intersect $\Lambda$. (c) A closed line, $\Lambda$, and points $A$ and $B$ outside the surrounded area. $\Gamma_{c}$ is a contour that intersects $\Lambda$ two times and $\Gamma_{s}$ is a contour that surrounds it.

change in the order of the states [see Refs. 37(a) and 37(b) where a similar situation is analyzed while studying charge transfer for the $\left(\mathrm{H}+\mathrm{H}_{2}\right)^{+}$system]. Thus, if at point $A$ state $1 A^{\prime \prime}$ is above $1 A^{\prime}$ we find, following contour $\Gamma_{c}$, that at $B 1 A^{\prime \prime}$ is below $1 A^{\prime}$. Moving along a contour $\Gamma_{c}$ that crosses $\Lambda$ twice leaves the order of the states unchanged [see Fig. 6(c)]. We return now to the above mentioned questions and we start by discussing (i): If $\Lambda$ is an infinite line then the order of states can be affected even in a situation where the test hydrogen is at an infinite separation from the $\mathrm{CH}_{2} \mathrm{NH}$ radical. However at large distances the hydrogen has no influence on the radical (and vice versa) and therefore cannot affect the states of the radical. In other words no contours of the kind $\Gamma_{c}$ can be found at the infinite separation region, which implies that $\Lambda$ is a finite line. Next we discuss (ii). Since $\Lambda$ is a finite line, it can be either an open or a closed line. In order to be able to follow the forthcoming analysis we refer again to Fig. 6 in which are presented several possibilities. In Fig. 6(a) is shown an open line, $\Lambda$, and two contours $\Gamma_{c}$ and $\Gamma_{s}$ that connect $A$ and $B$. From the earlier discussion we find that moving along these two contours is expected to yield, physically, unacceptable results; namely, moving along $\Gamma_{c}$ changes the order of states but moving along $\Gamma_{s}$ leaves them unchanged. A different situation is encountered when $\Lambda$ is a closed line as given in Figs. 6(b) and 6(c). In the case of Fig. 6(b) any contour from $A$ to $B$ is 
a $\Gamma_{c}$-contour and therefore affects, once, the order of the states. The opposite is true for the case described in Fig. 6(c); namely, any contour from $A$ to $B$, whether intersecting, $\Lambda$, or not leaves the order of the states unchanged. To summarize this analysis we say that open lines, in contrast to closed lines, lead to unphysical situations and therefore cannot exist in molecular systems.

(3) In the present study we also encounter an uncommon Berry-type phase which is $\sim 0$ instead of being an integer multiple of $\pi$. Such a result is expected when a seam and the closed circular contour are located in the same plane so that the contour intersects the seam instead of surrounds it (by leaving the plane). In Refs. 37(a) and 37(b) we analyzed similar situations and showed that each intersection between the contour and such a seam forms for the corresponding (in our present case, the angular) component of the NACT, a narrow function that in the limiting case becomes a Dirac $\delta$-function, namely, $(\pi / 2) \delta\left(\varphi-\varphi_{s}\right)$, which yields upon integration (over $\varphi$ ) the ADT angle $\gamma=\pi / 2$. Here $\varphi_{s}$ is the $\varphi$-value at the intersection. The corresponding $n u$ merical integrations along the closed contour revealed in our case that at the vicinity of the first intersection, the value of the ADT angle $\gamma$ is approximately $+\pi / 2$ and at the second it is approximately $-\pi / 2$. As a result, the final Berry phase is approximately zero. This outcome, although somewhat unexpected, is in agreement with other studies of similar situations. ${ }^{37,39,40}$

(4) In order to strengthen the idea that this line of cis is indeed a seam, we managed also to calculate the angular NACTs along a contour, which is slightly tilted away from the HC-NHH plane. In this way the contour, instead of intersecting the line of cis, surrounds it. The results are presented in Fig. 2(c). It is clearly seen that in contrast to the two previous functional shapes this one is characterized by two positive peaks. Calculating the Berry phase for this case yields the value of 3.124 , a value close to $\pi$. This issue will be further discussed in a forthcoming publication.

Finally we would like to refer to two aspects due to this study, a physical aspect and a numerical one:

(a) From the physical point of view this new type of seams is expected to guarantee a much more efficient decay process from an upper adiabatic state to a lower one. The existence of such lines of cis-instead of isolated cis (as exhibited by other polyatomic $(n>3)$ molecules such as $\mathrm{HNCO}$ [Ref. 8(b)] or $\mathrm{C}_{2} \mathrm{H}_{2}$ [Refs. 4(b) and 4(c) - - may enhance significantly the transition rate between the two PESs.

(b) From a computational point of view this finding may affect future quantum mechanical dynamical studies of systems of this type. Thus, if such a degeneracy line is located in the path of a wave packet which moves from the upper PES to the lower one (as seen in Fig. 5) we may perform, instead of a (diabatic) two-state calculation, a diabatic single-state calculation and still obtain accurate results. In fact such a calculation is on its way, and we hope to report the outcomes in the near future. In this respect we would like to mention again the first theoretical study on this system. ${ }^{24}$ In addition to determining the position of the point of degeneracy (for the same configuration), Dunn and Morokuma ${ }^{24}$ also calculated the two adiabatic potential energy curves along the $\mathrm{N}-\mathrm{H}$ dissociation channel, which are presented in their Fig. 2 as dashed lines. A good qualitative fit is achieved between their potential curves and our PESs.

\section{ACKNOWLEDGMENTS}

M.B. and C.L. acknowledge the US-Israel Bi-national Science Foundation for partly supporting this study. Á.V. acknowledges OTKA Grant No. T067923 and the computational resources provided by the John-von-Neumann Institute, Research Centre Juelich (Project ID ehu01). I.B. and C.L. acknowledge the partial support of the James Franck Binational German-Israeli Program in Laser-Matter Interaction and the Israel Science Foundation founded by The Israel Academy of Science and Humanities.

${ }^{1}$ M. Baer, Beyond Born Oppenheimer: Electronic non-Adiabatic Coupling Terms and Conical Intersections (Wiley, Hoboken, NJ, 2006).

${ }^{2}$ in particular, see (a) M. S. Child, Adv. Chem. Phys. 124, 1 (2002); (b) S. Adhikari and G. D. Billing, ibid. 124, 143 (2002); (c) R. Englman and A. Yahalom, ibid. 124, 197 (2002); (d) A. Kuppermann and R. Abrol, ibid. 124, 323 (2002); (e) G. A. Worth and M. A. Robb, ibid. 124, 355 (2002). ${ }^{3}$ A. Kuppermann, in Dynamics of Molecules and Chemical Reactions, edited by R. E. Wyatt and Z. H. Zhang (Marcel, New York, 1996), p. 411; R. Baer, D. M. Charutz, R. Kosloff, and M. Baer, J. Chem. Phys. 105, 9141 (1996); S. Adhikari and G. D. Billing, ibid. 111, 40 (1999).

${ }^{4}$ (a) G. J. Halász, Á. Vibók, A. M. Mebel, and M. Baer, Chem. Phys. Lett. 358, 163 (2002); (b) Á. Vibók, G. J. Halász, and M. Baer, ibid. 413, 226 (2005); (c) G. J. Halász, Á. Vibók, S. Suhai, and M. Baer, J. Chem. Phys. 127, 244101 (2007).

${ }^{5}$ G. J. Halász, Á. Vibók, R. Baer, and M. Baer, J. Phys. A: Math. Theor. 40, F267 (2007).

${ }^{6}$ G. J. Halász, Á. Vibók, D. K. Hoffman, D. J. Kouri, and M. Baer, J. Chem. Phys. 126, 154309 (2007).

${ }^{7}$ Conical Intersections: Electronic Structure, Dynamics and Spectroscopy, edited by W. Domcke, D. R. Yarkony, and H. Köppel (World Scientific, Singapore, 2004). In particular see: (a) D. R. Yarkony, p. 41; (b) H.. Köppel, p. 175; (c) R. de Vivie-Riedle and A. Hofmann, p. 803.

${ }^{8}$ (a) R. G. Sadykov and D. R. Yarkony, J. Chem. Phys. 109, 20 (1998); (b) D. R. Yarkony, ibid. 114, 2614 (2001).

${ }^{9}$ E. R. Davidson, J. Am. Chem. Soc. 99, 397 (1977).

${ }^{10}$ E. S. Kryachko, Adv. Quantum Chem. 44, 119 (2003).

${ }^{11}$ P. Barragan, L. F. Errea, A. Macias, L. Mendez, A. Riera, J. M. Lucas, and A. Aguilar, J. Chem. Phys. 121, 11629 (2004); M. B. Sevryuk, L. Y. Rusin, S. Cavalli, and V. Aquilanti, J. Phys. Chem. A 108, 8731 (2004); R. Englman and T. Vertesi, Phys. Lett. A 354, 196 (2006).

${ }^{12}$ S. Amaran and S. Kumar, J. Chem. Phys. 128, 154325 (2008); S. Amaran, S. Kumar, and H. Koeppel, ibid. 128, 124305 (2008); S. GomezCarrasco, A. Aquado, M. Paniaqua, and O. Roncero, ibid. 125, 104105 (2006).

${ }^{13}$ C. Hu, H. Hirai, and O. Sugino, J. Chem. Phys. 128, 144111 (2008); 127, 064103 (2007).

${ }^{14}$ E. Abrahamsson, G. C. Groenenboom, and R. V. Krems, J. Chem. Phys. 126, 184309 (2007); V. A. Mozhayskiv, D. Babikov, and A. I. Krylov, ibid. 124, 224309 (2006).

${ }^{15}$ M. Barbatti, S. Belz, M. Leibscher, H. Lischka, and J. Manz, Chem. Phys. 350, 145 (2008).

${ }^{16}$ P. Puzari, B. Sarkar, and S. Adhikari, J. Chem. Phys. 121, 707 (2004); B. Sarkar and S. Adhikari, ibid. 124, 074101 (2006); P. O. Godsi, C. R. Evenhuis, and M. Collins, ibid. 125, 164321 (2006).

${ }^{17}$ M. Kreglewski, J. Mol. Spectrosc. 133, 10 (1989).

${ }^{18}$ J. V. Michael and W. A. Noyes, J. Am. Chem. Soc. 85, 1228 (1963). 
${ }^{19}$ M. N. R. Ashfold, R. N. Dixon, M. Kono, D. H. Mordaunt, and C. V. L. Reed, Philos. Trans. R. Soc. London, Ser. A 355, 1659 (1997).

${ }^{20}$ G. C. G. Waschewsky, D. C. Kitchen, P. W. Browning, and L. J. Butler, J. Phys. Chem. 99, 2635 (1995).

${ }^{21}$ R. Marom, U. Zecharia, S. Rosenwaks, and I. Bar, Chem. Phys. Lett. 440, 194 (2007).

${ }^{22}$ R. Marom, U. Zecharia, S. Rosenwaks, and I. Bar, Mol. Phys. 106, 213 (2008).

${ }^{23}$ R. Marom, U. Zecharia, S. Rosenwaks, and I. Bar, J. Chem. Phys. 128, 154319 (2008).

${ }^{24}$ K. M. Dunn and K. Morokuma, J. Phys. Chem. 100, 123 (1996).

${ }^{25}$ E. Kassab, J. T. Gleghorn, and E. M. Evleth, J. Am. Chem. Soc. 105, 1746 (1983)

${ }^{26}$ H. A. Jahn and E. Teller, Proc. R. Soc. London, Ser. A 161, 220 (1937)

${ }^{27} \mathrm{R}$. Englman, The Jahn-Teller Effect in Molecules and Crystals (WileyInterscience, New York, 1972).

${ }^{28}$ M. Baer, A. M. Mebel, and R. Englman, Chem. Phys. Lett. 354, 243 (2002).
${ }^{29}$ MOLPRO, a package of ab initio programs designed by H.-J. Werner and P. J. Knowles, R. Lindh, F. R. Manby, M. Schütz et al.

${ }^{30}$ M. Born and J. R. Oppenheimer, Ann. Phys. 84, 457 (1927).

${ }^{31}$ M. Born and K. Huang, Dynamical Theory of Crystal Lattices (Oxford University Press, New York, 1954), Chap. IV.

${ }^{32}$ M. Baer, Chem. Phys. Lett. 35, 112 (1975).

${ }^{33}$ M. Baer and R. Englman, Mol. Phys. 75, 293 (1992).

${ }^{34}$ D. R. Yarkony, J. Chem. Phys. 105, 10456 (1996).

${ }^{35}$ M. Baer, J. Chem. Phys. 107, 2694 (1997).

${ }^{36}$ M. Baer, S. H. Lin, A. Alijah, S. Adhikari, and G. D. Billing, Phys. Rev. A 62, 032506 (2000).

${ }^{37}$ (a) Z. H. Top and M. Baer, Chem. Phys. 25, 1 (1977); (b) M. Baer, G. Niedner, and J. P. Toennies, J. Chem. Phys. 91, 4169 (1989); (c) G. D. Billing, M. Baer, and A. M. Mebel, Chem. Phys. Lett. 372, 1 (2003).

${ }^{38}$ C. A. Mead, J. Chem. Phys. 70, 2276 (1979).

${ }^{39}$ Y. Lyanda-Geller, Phys. Rev. Lett. 71, 657 (1993).

${ }^{40}$ T. Vertesi and R. Englman, J. Phys. B 41, 025102 (2008). 